\title{
Cost effectiveness of adding CMR to evaluation of suspected coronary ischaemia
}

\author{
Deirdre F Waterhouse*, Roisin B Morgan, Rory OHanlon \\ From 15th Annual SCMR Scientific Sessions \\ Orlando, FL, USA. 2-5 February 2012
}

\section{Summary}

To estimate the cost and diagnostic implications of using CMR alone instead of conventional TTE/TMET work-up to guide patient selection for angiography. Healthcare costs were derived from VHI's hospital billing system.

\section{Background}

Patient selection for coronary angiography traditionally relies on clinical assessment, treadmill exercise testing (TMET) and transthoracic echocardiography (TTE). Cardiac Magnetic Resonance (CMR) is a relatively novel imaging study, which provides excellent non-invasive assessment of myocardial perfusion and is useful in risk stratification of patients with suspected coronary artery disease (CAD).

\section{Methods}

Consecutive patients referred to our center for CMR evaluation within a 12-month period (january 2010 to january 2011) were enrolled. This population consisted of patients with suspected CAD who were candidates for invasive coronary angiography but first underwent stress $\mathrm{cmr}$ for further risk stratification.

\section{Results}

83 Patients (64 male, 19 female) with suspected CAD underwent CMR. 15.4\% had ischaemic features on TMET and $47 \%$ had evidence of territorial ischaemia on TTE. On CMR evaluation, $38.6 \%$ of these were found to have definite CAD. Interestingly, $18 \%$ of patients had significant CAD on CMR despite no evidence of ischaemia on TMET and TTE and would not have undergone angiography based on conventional assessment.

In 16 cases (19.3\%), planned angiography based on abnormal TTE/TMET was avoided by CMR which excluded a diagnosis of CAD. Furthermore, non-ischaemic causes of cardiac symptoms were discovered on $8.4 \%$ of CMR which were undetected on conventional workup.

The use of CMR as first line investigation in assessment of suspected coronary ischaemia would have avoided TMET in $31.3 \%$, TTE in $98.7 \%$ and angiography in $25.3 \%$. This would represent a total saving of $€ 18722$, or $€ 226$ per patient.

\section{Conclusions}

This study demonstrated that a CMR-only approach is the most cost-effective diagnostic strategy for evaluation of CAD. CMR imaging allows accurate selection of patients for invasive management, avoiding unnecessary procedures. CMR was as useful as angiography in guiding revascularisation and is superior to TMET/TTE in detecting ischaemia.

\section{Funding}

None to disclose.

Published: 1 February 2012

doi:10.1186/1532-429X-14-S1-P40

Cite this article as: Waterhouse et al.: Cost effectiveness of adding CMR

to evaluation of suspected coronary ischaemia. Journal of Cardiovascular Magnetic Resonance 2012 14(Suppl 1):P40.

Ronan Cahill, CMR Unit Blackrock Clinic, Dublin, Ireland

(c) 2012 Waterhouse et al; licensee BioMed Central Ltd. This is an open access article distributed under the terms of the Creative 